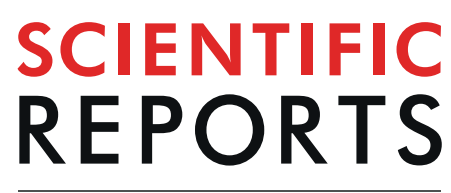

natureresearch

Check for updates

\title{
OPEN Micro-Nano formulation of bile-gut delivery: rheological, stability and cell survival, basal and maximum respiration studies
}

\begin{abstract}
Susbin Raj Wagle $\mathbb{D}^{1}{ }^{1}$, Daniel Walker ${ }^{1}$, Bozica Kovacevic ${ }^{1}$, Ahmed Gedawy ${ }^{1}$, Momir Mikov ${ }^{2}$, Svetlana Golocorbin-Kon ${ }^{3}$, Armin Mooranian $\mathbb{1 B}^{1}$ \& Hani AI-Salami ${ }^{1 凶}$

Probucol (PB) is a drug that exhibits significant hydrophobicity and substantial intra and inter individual variability in oral absorption, with a miniature bioavailability and complex three compartmental pharmacokinetic modelling due to its high lipid affinity, low stability and high octanol to water partition coefficient. Multiple attempts to formulate PB have not produced satisfactory stable matrices, drugrelease profile or rheological flow properties for optimum manufacturing conditions, and with positive and none toxic biological effects. Lithocholic acid (LCA) has recently shown to optimise formulation and cell uptake of drugs. Hence, the aim of this study was to design new PB delivery system, using LCA, and examine its morphology, rheology, stability, and cellular effects. PB was formulated with LCA and sodium alginate (PB-LCA-SA) using various microencapsulation methodologies, and best formulation was investigated in vitro and ex vivo. Using our lonic Gelation Vibrational Jet flow technology, PB-LCA-SA microcapsules showed good stability and significantly enhanced cell viability, cellular respiration, and reduced inflammation suggesting potential LCA applications in PB delivery and biological effects.
\end{abstract}

More than $40 \%$ of drugs are lipophilic and exhibit poor water solubility and low bioavailability, despite favourable pharmacological activity ${ }^{1}$. A common and promising approach to improve the drug's low water solubility and bioavailability is using encapsulation technology as well as formulation strategies to design new matrices where drugs can be incorporated, and delivered efficiently in the body, after oral administration ${ }^{2-5}$. Artificial cell microencapsulation (ACM) is widely used to microencapsulate and deliver lipophilic drugs that show poor dissolution and absorption kinetics and low bioavailability ${ }^{6-8}$. Microencapsulation of lipophilic drugs is one of the most promising applications in diabetes therapy and it encompasses the use of polymers/copolymers mixture to encapsulate and engulf a drug or a therapeutic entity conferring improved stability and targeted delivery properties $^{9-11}$. The technology has been widely researched to optimise delivery and stability of therapeutics including drugs, viable cells and proteins ${ }^{12-15}$.

Diabetes Mellitus (DM) is a disease classified generally into two forms, type 1 diabetes (T1D) and type 2 diabetes (T2D) ${ }^{16}$. Millions of people are globally affected by DM and it is anticipated to reach more than 438 million by 2030 , which is roughly $8 \%$ of the adult population ${ }^{17,18}$. Every region in the world has been affected by the disease, and its incidence is high in countries such as Australia, Europe, North America and countries undergoing westernization including India and China ${ }^{19}$. In 2016, it was estimated that total diabetes-related global costs more than 100 billion USD, which is more than $12 \%$ of global combined health expenditure ${ }^{18,20}$.

Insulin treatment is widely used in diabetes therapy, with all T1D patients, and in T2D more than one-third of T2D patients using insulin. Insulin is produced by pancreatic $\beta$-cells located in the islets of Langerhans. One of the common symptoms associated with T2D development and progression is chronic inflammation of $\beta$-cells as well as high levels of low-density lipoproteins (LDL), free radicals and oxidants, which have been connected to exacerbation and worsening of diabetes-associated complications ${ }^{21}$. Recent studies have shown that patients

\footnotetext{
${ }^{1}$ Biotechnology and Drug Development Research Laboratory, School of Pharmacy and Biomedical Sciences, Curtin Health Innovation Research Institute, Curtin University, Perth, Western Australia, Australia. ${ }^{2}$ Department of Pharmacology, Toxicology and Clinical Pharmacology, Faculty of Medicine, University of Novi Sad, Novi Sad, Serbia. ${ }^{3}$ Department of Pharmacy, University of Novi Sad, Novi Sad, Serbia. ${ }^{\circledR}$-mail: hani.al-salami@curtin.edu.au
} 
with lipid-disorders such as hypercholesterolemia are three times more likely to develop T2D and there is a significant association between T2D and cardiovascular disease ${ }^{22,23}$. Published studies have also shown that significant inflammation and damage of pancreatic $\beta$-cell plays a major role in diabetes and cardiovascular disease development and progression, particularly since $\beta$-cells have limited defence against free radicals, oxidants and LDL-associated cellular toxicity ${ }^{24,25}$. Accordingly, new or adjunct antidiabetic drugs should exhibit anti-atherosclerotic, antilipidemic, antioxidant, anti-free radical and $\beta$-cell protective effects.

Probucol is a drug marketed for hypercholesteremia and remains widely prescribed in some countries such as China, and probably to a lesser extent in other countries such as Japan and India ${ }^{26}$. Despite its potent and powerful effects in lowering low density lipoprotein (LDL), probucol oral delivery has many challenges which resulted in its withdrawal from many countries including Australia and the USA. Probucol has high lipophilicity, poor water solubility, complex 3-compartmental pharmacokinetic modelling with variable and miniature bioavailability resulting in inconsistent oral uptake and severe side effects in some patients ${ }^{26,27}$. To make matters worse, probucol current oral dosage form, a tablet, has been used since its initial formulation development in the 1960s and remains under developed. Accordingly, in order to overcome challenges in probucol oral uptake in its current dosage form, new oral delivery matrices using cutting-edge technologies are needed. Such technologies can include microencapsulation with new excipients, that have demonstrated powerful oral targeted delivery, permeation enhancement properties and substantial consistent release profiles ${ }^{6,28}$.

Bile acids (BAs) are endogenously produced in animals and humans and notionally are known to facilitate food digestion, and vitamin absorption from the gastrointestinal tract. Due to their amphiphilic nature, recent studies have suggested potential applications of BAs in the oral delivery of lipophilic drugs, as formulation excipients, and permeation enhancers ${ }^{29,30}$. Common primary bile acids are cholic acid and chenodeoxycholic acid, while secondary and tertiary bile acids are deoxycholic acid, lithocholic acid, taurocholic acid, and ursodeoxycholic acid. Overall, there are more than 100 types of bile acids although their ratios in the gut-contents, blood, and tissues vary widely depending on species and health status ${ }^{31}$. Several studies in our lab, as well as others have attempted to design new and powerful nano and micro based delivery systems for probucol oral uptake, using some bile acids as well as other excipients' combinations $s^{8,10,32-34}$. However, in preclinical studies, probucol absorption remains variable, and not ideal ${ }^{30}$. The bile acid lithocholic acid (LCA) has recently shown to optimise formulation and cell delivery ${ }^{35}$. It is one of the most prominent secondary bile acids and has recently displayed the potential application in the oral delivery of anticancer drugs ${ }^{36}$. It can self-assemble to develop nanostructure, and it has unique amphiphilic properties, high structural rigidity and reasonable biocompatibility ${ }^{37}$. LCA has anti-inflammatory properties and has shown positive effects on inflammatory bowel diseases ${ }^{38}$.

In previously published work, our lab demonstrated the potential application of bile acids, either alone or in combination with anti-diabetic drugs and polymers by forming microcapsules ${ }^{8,10,39}$. To date, ideal microcapsules for oral delivery of probucol would exhibit significant targeted properties, proper self-assemble profile, and unique amphiphilic properties with structural integrity and excellent biocompatibility, however this remains elusive and inconsistent. Thus, the objective of study is to examine the suitability, stability and biological activity of newly formed probucol microcapsules without and with lithocholic acid.

\section{Results and Discussion}

Morphology, size analysis and chemical characterization of microcapsules. Figure 1(A) shows that optical microscopy (OM) examination of both formulations, PB-SA (i) and PB-LCA-SA (ii) maintained their spherical, round shape and similar size. OM results suggest that the incorporation of LCA did not significantly change microcapsules' topography, uniformity, shape, size, or morphology. SEM (scanning electron microscope) results show well defined spherical shape microcapsule, Fig. 1B (i) and Fig. 1C (i). Microcapsules surface were rough, and Fig. 1B (ii), (iii), and (iv) and Fig. 1C (ii), (iii), and (iv) show that PB-SA and PB-LCA-SA microcapsules presented solid structure with small granules on the surface, which suggests that the incorporation of LCA into PB-SA formulation did not affect microcapsules morphology. Energy dispersive X-ray spectrometry (EDS) (Fig. 2) was used to analyse the element components present on the outer layer of the microcapsules. Figure 2A shows the surface analysis of PB-SA microcapsules and Fig. 2B shows the surface analysis of PB-LCA-SA microcapsules at different sites with their corresponding spectra. Three different locations were randomly chosen. These Figs. 2a-f represent the crystal deposition on microcapsules' surface. Spectral analysis displays a high level of the sulphur atom in both formulations which indicate $\mathrm{PB}$ deposition ${ }^{40}$. Other elements such as $\mathrm{O}$ and $\mathrm{C}$ are anticipated to be present on the surface of microcapsules because these elements were part of the polymer and encapsulation processes deployed ${ }^{41,42}$. Figure 2 suggests that incorporation of LCA does not compromise or significantly alter the PB distribution within the microcapsules' layers and potentially, PB release profile.

Rheological, thermal and chemical profiles. For control and test formulations, Table 1 and Figs. 3A-B provide the rheological profiles, while Fig. $3 \mathrm{C}$ and Table 2 provide thermal and the chemical profiles.

Torque profile was elevated by higher speed of mixing, and LCA incorporation did not change that, however, at highest speed, Torque remained lower when LCA was present, suggesting an alteration to the turning-power ability and resistance of the PB-SA formulation as a result of LCA incorporation. Similarly, both control and test formulations exhibited Non-Newtonian shear-thinning rheological behaviour with LCA incorporation resulting in sharper decrease in viscosity associated with consistent increase in shear stress. As mixing speed increases, shear rate of both formulation showed consistent increase, supporting a steady rheological profile with or without LCA. In addition, during mixing, both formulations form quick circular motions away from the site of centripetal force at an increasing speed of the stirring rod suggesting both formulations acted in a non-Weissenberg behaviour, which is consistent with previous studies ${ }^{43,44}$. These results provide useful new knowledge that can contribute to design of new formulation systems via demonstrating the effect of selective concentrations of LCA on the formulation rheology and force (and dimentions via Torque) needed for capsule fabrication and stability profiles. 


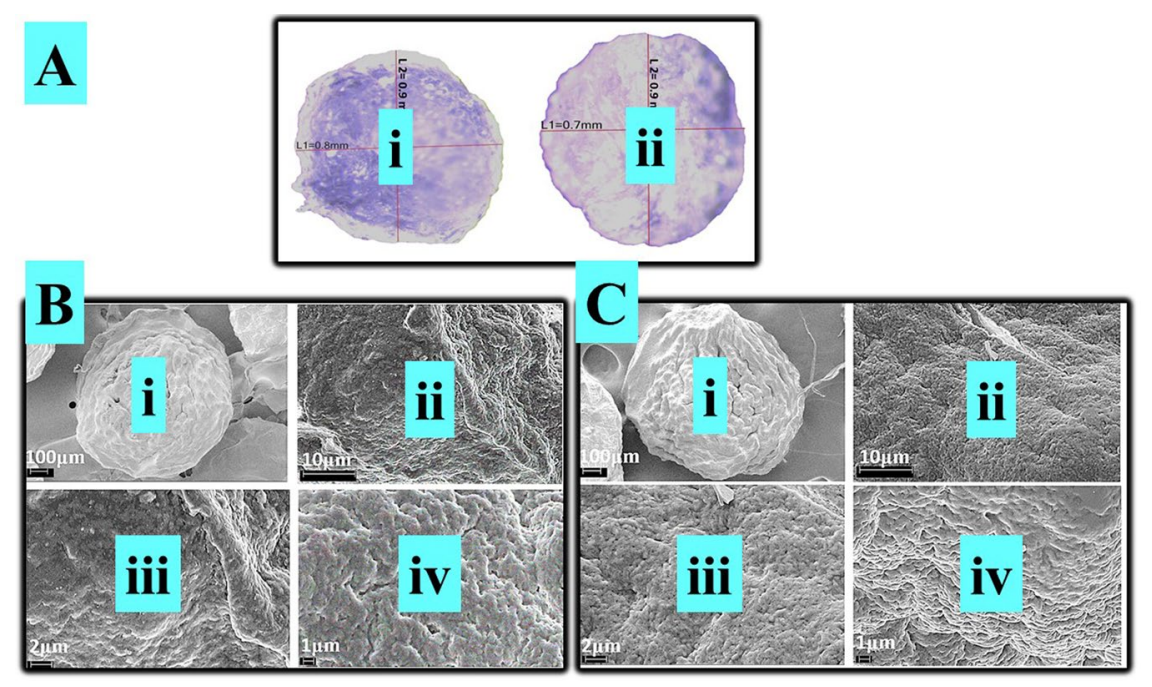

Figure 1. (A) Optical microscopy of PB-SA (i) and PB-LCA-SA (ii) microcapsules. (B)Scanning electron micrographs of PB-SA revealing microcapsules morphology and surface topography took at different magnifications, (i) $100 \mu \mathrm{m}$ scale (ii) Surface morphology at $10 \mu \mathrm{m}$ scale. (iii) $2 \mu \mathrm{m}$ scale (iv) $1 \mu \mathrm{m}$ scale. (C) Scanning electron micrographs of PB-LCA-SA and surface topography taken at different magnifications, (i) $100 \mu \mathrm{m}$ scale (ii) Surface morphology at $10 \mu \mathrm{m}$ scale. (iii) $2 \mu \mathrm{m}$ scale (iv) $1 \mu \mathrm{m}$ scale. PB- probucol; SA- sodium alginate; LCA-lithocholic acid.

The differential scanning calorimetry (DSC) thermal peaks provide information on thermal capacity and excipient-excipient and excipient-drug compatibilities. Thermal peak of $\mathrm{PB}$ powder is expected around $130^{\circ} \mathrm{C}$ region, which correlates to $\mathrm{PB}$ melting temperature, while thermal peak of LCA powder is expected around $200^{\circ} \mathrm{C}$ region, which correlates to LCA melting temperature. Thermal peak of SA powder is expected around $190^{\circ} \mathrm{C}$ region, which correlates to SA melting temperature ${ }^{45}$. With the LCA and SA sharing similar melting temperatures, their peaks were expected to unit, which was observed in Fig. 3C, with potential amorphous phase formation. Results showed that the addition of LCA did not significantly alter the formulation heat capacity and did not result in significant changes of heat emission, or appearance of new peaks but slight endothermic peak shift ${ }^{46}$. Hence, during pre and post microencapsulation processes, the observed endothermic shift in the melting point of PB-SA, and PB-LCA-SA may be influenced by the ionic interaction between the ingredients constituting the microcapsules, alterations in the crystallinity, plasticization, and polymorphism of SA without compromising the overall stability ${ }^{47}$. Thermal compatibility of PB with other bile acids has been published ${ }^{10}$, supporting our finding. In our study, DSC analyses were complemented with FTIR (Fourier transform infrared) studies, to describe PB, LCA and SA stability and compatibility profiles.

FTIR studies are commonly used to examine the vibrational frequency levels of different functional groups present within the molecules or between molecules, which reflects chemical bond alteration and stability ${ }^{48}$. FTIR spectra were used to examine chemical stability and compatibility of PB with the polymer SA and the bile acid LCA pre and post-microencapsulation. PB, SA and LCA powders were analysed individually, combined, then pre and post encapsulation. The $\mathrm{PB}$ powder spectra analysis displayed three different characteristics peaks at 2955.16, 1421 and $1308 \mathrm{~cm}^{-1}$ which confirms the presence of its functional groups, and is in line with other studied (Table 2) ${ }^{10,48,49}$. Likewise, the SA powder analysis exhibited predominant $\mathrm{O}-\mathrm{H}$ stretching intense peak at $3253 \mathrm{~cm}^{-1}$ and three medium intensity peaks at 1569,1405 and $1025 \mathrm{~cm}^{-1}$ which is aligned with other studies ${ }^{10,49}$, while the spectra of LCA powder revealed distinct peaks at 3282, 2925, 1701 and $1033 \mathrm{~cm}^{-1}$ indicating the presence of LCA (Table 2). The combined mixtures PB-LCA-SA shows the distinct peaks of all compounds without any interference, alternations or dilution which suggests chemical compatibility pre-microencapsulation. PB-SA (control) microcapsules analysis reveals characteristic peaks at 1591, 1416, 1300 and $1026 \mathrm{~cm}^{-1}$ which correspond to $\mathrm{PB}$ and SA analysis (Table 2). Similar peaks were found after LCA addition (test) and the peak at $1712 \mathrm{~cm}^{-1}$ $(\mathrm{C}=\mathrm{O})$ confirms the presence of LCA. This result confirms an interaction between LCA and SA but not with PB as found in DSC results. A minor shift towards the right side of the PB peak was seen in both formulation that may have been brought out by SA or LCA. This interaction did not seem to affect PB peaks that remain present suggesting PB stability and excipient-compatibility (Table 2).

LCA incorporation into $\mathrm{PB}$ microcapsules did not seem to affect compatibility or stability profiles of the microcapsules, but may influence $\mathrm{PB}$ release profiles from the microcapsules (Fig. 4).

Microcapsule disintegration, drug release and dissolution studies. Figure 4 shows $\mathrm{PB}$ release from control (PB-SA) and test (PB-LCA-SA) microcapsules in gut-simulating media, at four $\mathrm{pH}(1.5,3,6$ and 7.8$)$ over 6 hours at $37^{\circ} \mathrm{C}$. The four (Fig. $4 \mathrm{~A}-\mathrm{D}$ ) conditions represent four different sites of PB absorption in the gastrointestinal tract ${ }^{10,50,51}$. PB microcapsules demonstrated significant dependence on the gut media rather than LCA incorporation. At lower $\mathrm{pH}$ (stomach \& duodenum), there was negligible release from control and test microcapsules, while at higher $\mathrm{pH}$ (ileum) there was small drug release, with no significant effects from LCA incorporation. At 


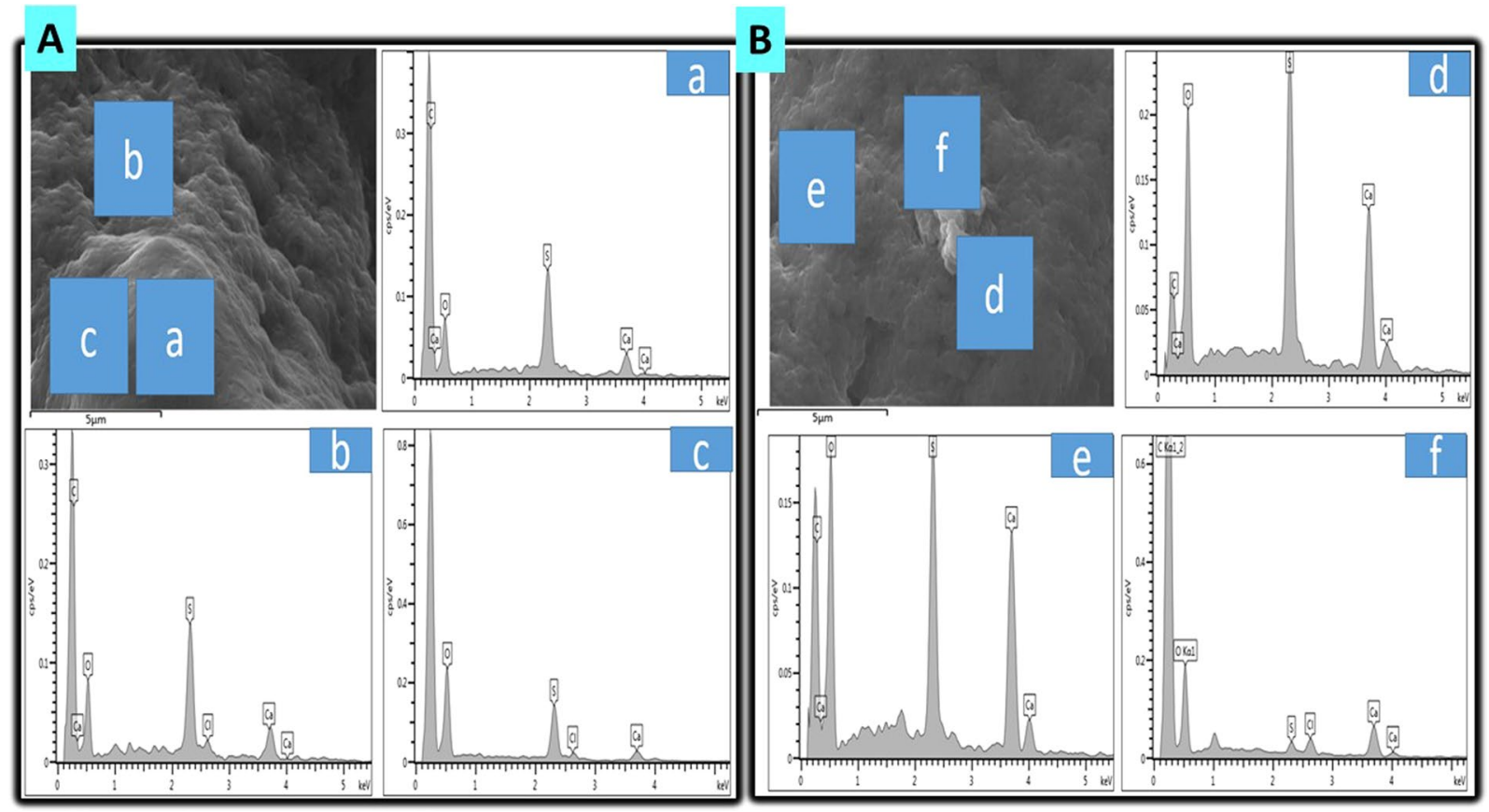

Figure 2. (A) EDS of PB-SA microcapsules and the corresponding elemental analysis (a-c). (B) EDS of PBLCA-SA microcapsules and the corresponding elemental analysis (d-f). PB- probucol; SA- sodium alginate; LCA-lithocholic acid.

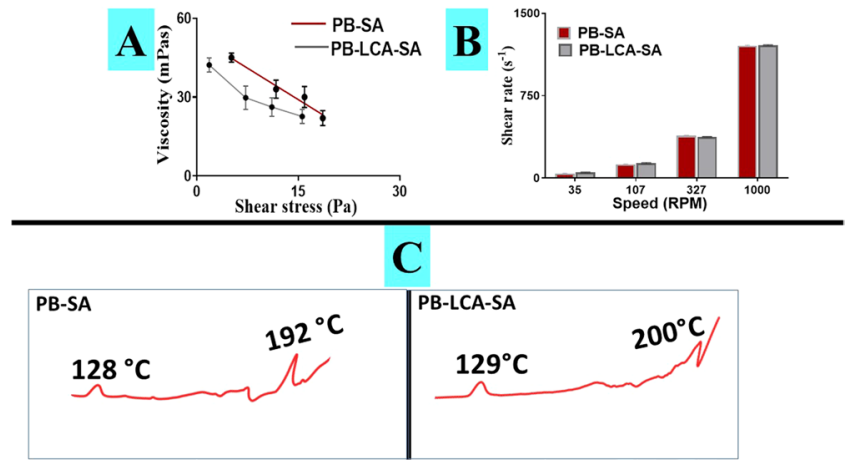

Figure 3. (A) Effect of shearing stress on viscosities, (B) Effect of speed on shear rate, (C) Dominant peaks observed by DSC analysis. PB- probucol; SA- sodium alginate; LCA-lithocholic acid.

\begin{tabular}{|l|l|l|l|}
\hline Formula code & Set Speed & RPM & Torque $(\mathbf{m N m})$ \\
\hline \multirow{4}{*}{ PB-SA } & 2 & 35 & $0.1 \pm 0.02$ \\
\cline { 2 - 4 } & 4 & 107 & $0.35 \pm 0.05$ \\
\cline { 2 - 4 } & 6 & 327 & $0.8 \pm 0.1$ \\
\cline { 2 - 4 } & 8 & 1000 & $1.5 \pm 0.03$ \\
\hline \multirow{3}{*}{ PB-LCA-SA } & 2 & 35 & BLOD \\
\cline { 2 - 4 } & 4 & 107 & $0.14 \pm 0.03$ \\
\cline { 2 - 4 } & 6 & 327 & $0.36 \pm 0.07$ \\
\cline { 2 - 4 } & 8 & 1000 & $0.68 \pm 0.17$ \\
\hline
\end{tabular}

Table 1. Rheological parameters for the formulations $(n=3$, mean \pm SEM, BLOD: Below Limit of Detection. PB- probucol; SA- sodium alginate; LCA-lithocholic acid.

high $\mathrm{pH}$ (cecum) there was substantial PB release with more than $50 \%$ within the first hour (Fig. 4D). The incorporation of LCA reduced PB release after 3 hours $(\mathrm{p}<0.05)$ and the overall release profiles of control and test remain similar at the end of the 6 hours experiment suggesting possibly a slightly more controlled PB release by 


\begin{tabular}{|l|l|l|}
\hline Formulation composition & FTIR spectra $\left(\boldsymbol{\lambda} \mathbf{~ c m}^{-1}\right)$ & Proposed functional groups \\
\hline PB powder & $2955.16,1421$ and 1308 & O-H, C-H, $=\mathrm{O}$ \\
\hline SA powder & $3253,1569,1405$ and 1025 & O-H, C=C, C-H, C-H \\
\hline LCA powder & $3282,2925,1701$ and 1033 & O-H, C-H, C=O, C-H \\
\hline PB-LCA-SA powder & $3270,2926,2854,1697,1600,1308$ and 1030 & O-H, C-H, C-H, C=O, C=C, S=O, C-H \\
\hline PB-SA microcapsules & $3340,2969,1591,1416,1300$ and 1026 & O-H, C-H, C=C, C-H, S=O, C-H \\
\hline PB-LCA-SA microcapsules & $3320,1593,1712,1417,1300$ and 1028 & O-H, C=C, C=O, C-H, C-H, C-H \\
\hline
\end{tabular}

Table 2. FTIR spectra of PB, SA and SA powder, PB-LCA-SA mixed powders and microcapsules. PB- probucol; SA- sodium alginate; LCA-lithocholic acid.

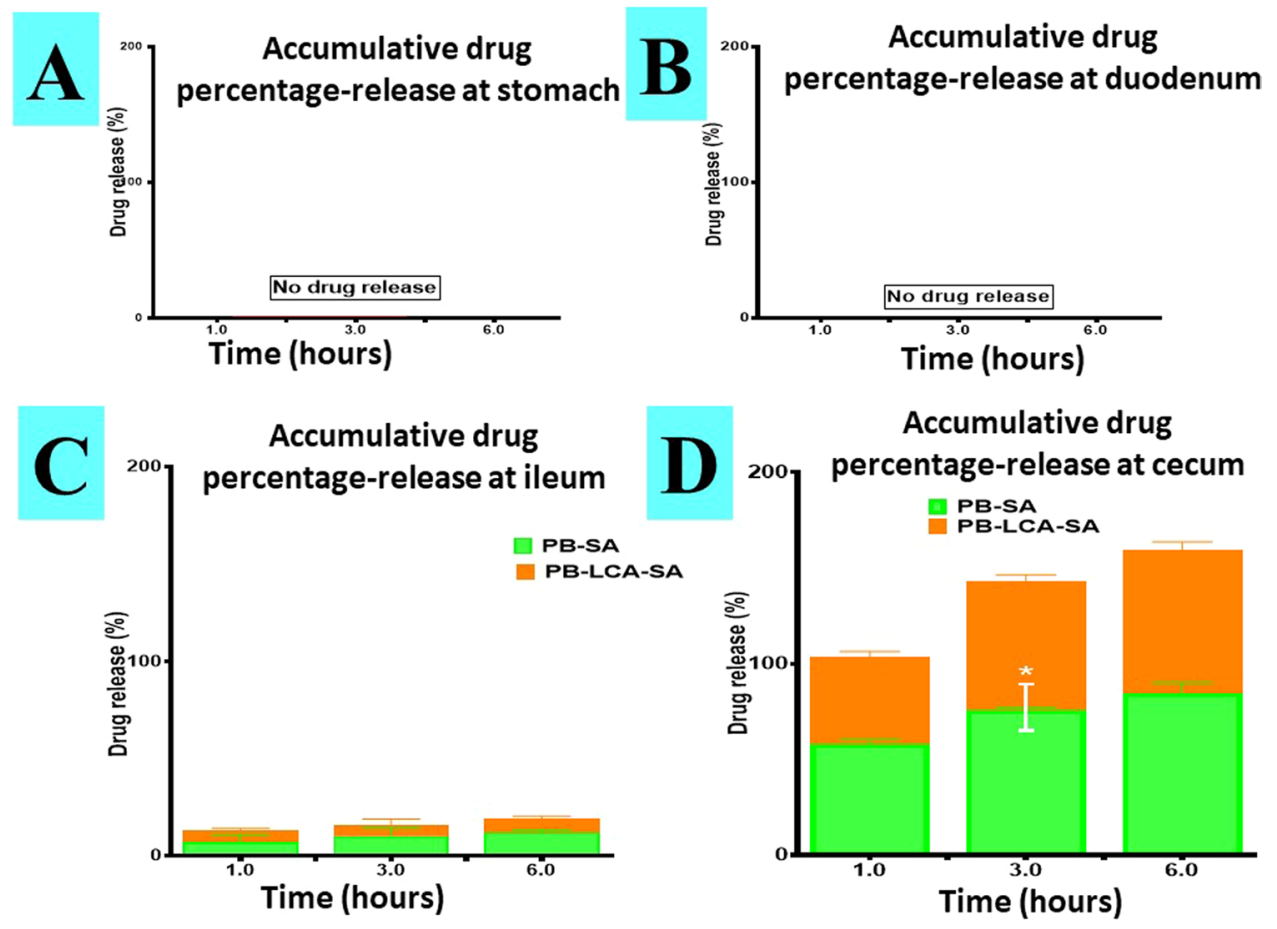

Figure 4. Microencapsules dissolution profiles in simulated gastric media at $\mathrm{pH} 1.5$ (A), $\mathrm{pH} 3(\mathbf{B}), \mathrm{pH} 6(\mathbf{C})$ and $\mathrm{pH} 7.8(\mathbf{D}) . \mathrm{N}=3$, mean \pm SD. PB- probucol; SA- sodium alginate; LCA-lithocholic acid. $* \mathrm{p}<0.05$.

LCA incorporation. At low $\mathrm{pH}$ (1.5, 3 and 6) alginic acid present on SA matrix results in shrinkage of alginate and thus encapsulated $\mathrm{PB}$ remained within core of the microcapsules but in higher $\mathrm{pH}(>6)$ due to rapid dissolution and solubilisation, alginic acid forms a soluble viscous layer ${ }^{52}$, resulting in the burst of PB. Similar studies have shown various and inconsistent effects of bile acids on drug release profiles, suggesting drug release from bile acid microcapsules can be formulation dependent $\mathrm{t}^{10,51}$.

Biological activity of PB-loaded microcapsules. Figure 5 shows cell viability at normoglycaemic $(5.5 \mathrm{mmol})$ and hyperglycaemic $(25 \mathrm{mmol})$ states (Fig. 5A), inflammatory (Fig. 5B-D) and bioenergetics (Fig. 5E-I) biological profiles of pancreatic $\beta$-cells line exposed for 48 hours to negative control (c; empty microcapsules), PB-SA and PB-LCA-SA microcapsules.

Figure 5A shows that at the hyperglycaemic state, PB-LCA-SA showed significantly higher cell viability compared with PB-SA which was higher than the sham control group, while at the normoglycaemic state, cell viability remained similar regardless of treatments. The higher cell viability, at the hyperglycaemic state as the result of treatments, suggests cellular protective effects brought about by PB, and enhanced by LCA, when encapsulated. In the literature, $\mathrm{PB}$ exhibits potent anti-oxidant, anti-inflammatory, and $\beta$ cells protection properties, and has shown to ameliorate hyperglycaemia-induced oxidative stress and inflammation ${ }^{53,54}$. Results suggest that LCA incorporation did not compromise cell viability, which might be due to modulation of the inflammatory and bioenergetics profiles. Figure 5(B-D) show that PB-SA exerted an anti-inflammatory effects via reducing levels of proinflammatory cytokines (IFN- $\gamma$ and IL-1 $\beta$ ) and enhancing levels of anti-inflammatory cytokine (IL-10), which is consistent with findings from Fig. 5A, and may relate directly to the controlled-release effects of LCA observed in Fig. 4D. Figure 5(E-I) show mitochondrial activity and cellular respiration of pancreatic $\beta$-cells at the normoglycaemic and hyperglycaemic states and results are in line with cell viability and inflammatory profiles and demonstrate significant activation of oxygen consumption rate (OCR), extracellular acidification rate 

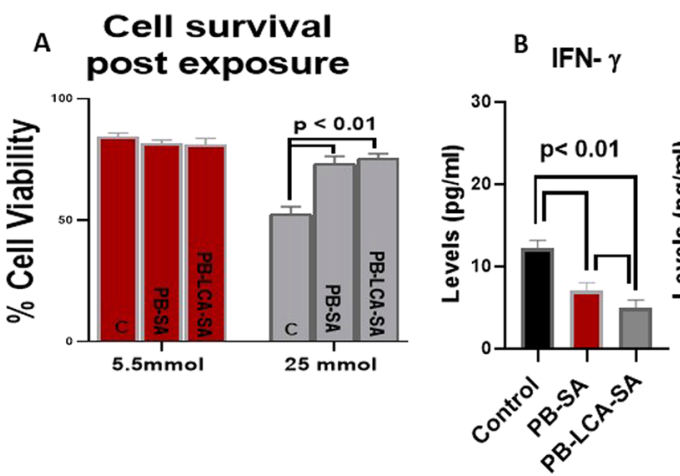

C

IL-1 $\beta$
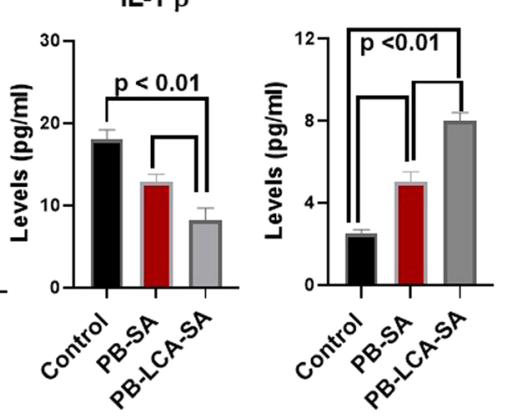

D IL-10

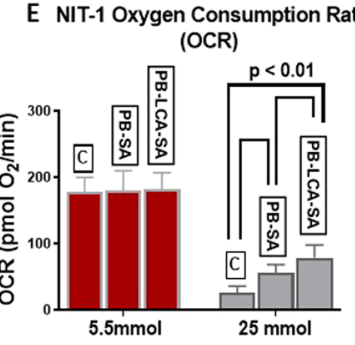

G

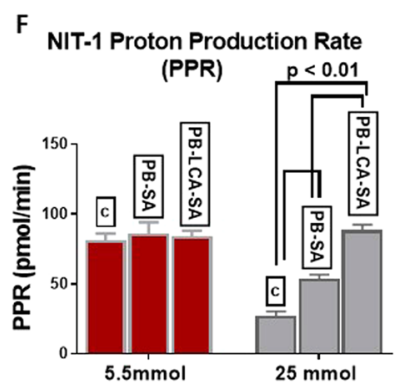

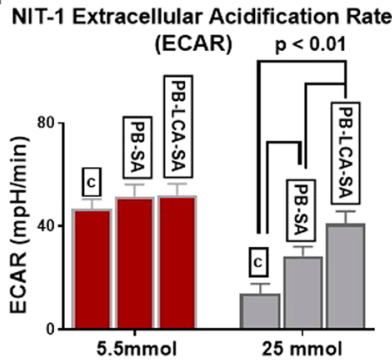
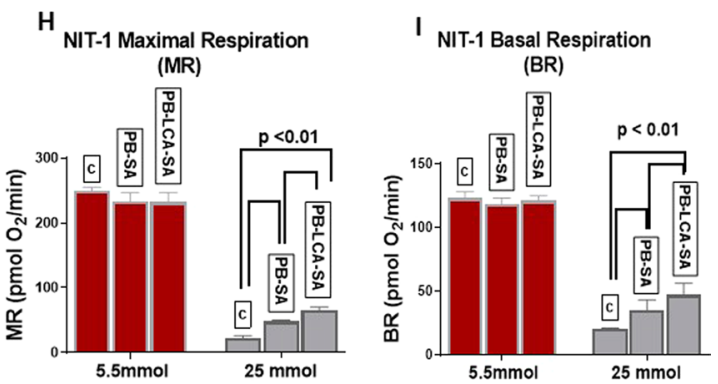

Figure 5. NIT-1 cell viability at $5.5 \mathrm{mmol}$ and $25 \mathrm{mmol}$ glucose (A), (B-D) NIT-1 level of inflammatory cytokines production, (E-I) bioenergetics parameters for NIT-1 beta cells at $5.5 \mathrm{mmol}$ and $25 \mathrm{mmol}$ glucose treated with PB-SA and PB-LCA-SA microcapsules. PB- probucol; SA- sodium alginate; LCA-lithocholic acid. Data values are mean $\pm \mathrm{SD}, \mathrm{N}=3 ; \mathrm{p}<0.01$.

(ECAR), proton production rate (PPR), maximal respiration $(\mathrm{MR})$ and basal respiration (BR) values when cells were exposed to PB-SA and PB-LCA-SA microcapsules compare with the hyperglycaemic control. Findings suggest that $\mathrm{PB}$ exerted a pancreatic $\beta$-cell protective effects possibly via reducing inflammation and enhancing biological bioenergetics, and these effects were optimised by LCA incorporation into PB microcapsules. At the normoglyacemic state, cellular bioenergetics remained similar regardless of treatments, while at the hyperglycaemic state, the treatments showed significant positive effects on biomarkers of cellular metabolism (PPR) and energetics (BR and $M R$ ) which were significantly and negatively affected at the hyperglycaemic state. For cellular respiration and acidification rates (OCR and ECAR), both treatments (PB-SA and PB-LCA-SA) showed consistent improvements to cellular bioenergetics and optimised biological functions at the hyperglycaemic states, which supports cellular biological improvements and viability results (Fig. 5). It is worth stating that significant elevation of bioenergetics biomarkers observed when cell treated with both microcapsules at the hyperglycaemic state, suggests that there is increased mitochondrial respiration and higher oxygen molecules serving as electron acceptors and assisting activity within the electron transport chain, with a proportional increase in oxidative phosphorylation, which is consistent with our previous studies ${ }^{55,56}$.

Biological measurements (Fig. 5) have shown positive and significant cell-protective, anti-inflammatory and pro-bioenergetics effects of the encapsulated PB and PB-LCA microcapsules on pancreatic $\beta$-cells. Compared with the literature, LCA on PB cellular effects are consistent with published data that have shown that bile acids as well as PB-loaded microcapsules enhanced beta-cell viability and functions ${ }^{8,57}$. In one of our recent studies, the bile acid ursodeoxycholic acid was incorporated with drug-containing microcapsules, and showed similar effects on drug release, and cell viability, and these effects were formulation-dependent ${ }^{31}$. Overall, this is the first study to elucidate LCA applications in PB-microcapsules', in the context of cell protection and diabetes treatment.

In conclusion, this study investigated the in vitro and ex vivo effects of LCA on PB-SA microcapsules in terms of capsules' morphology, rheological, thermal and chemical profiles, and biological effects on pancreatic $\beta$-cells. Results showed that the LCA did not compromise the pharmaceutical quality of the PB microcapsules, and it enhanced $\mathrm{PB}$ release profile as well as the biological effects at the hyperglycaemic state suggesting potential beneficial effects in diabetes therapy. The overall procedure along with the study results is presented in Fig. 6.

\section{Materials and Method}

Materials. Probucol (PB; 99.89\%) and sodium alginate (SA; 99\%) were obtained from Sigma-Aldrich, (St Louis, MO, USA) respectively. Lithocholic acid (LCA; $\geq 95 \%$ ) was purchased from Sigma- Aldrich, Co., USA. Calcium chloride dehydrate (98\%) was purchased from Scharlab S.L (Australia). All other required chemical and solvents were obtained from Merck and Co, and were of analytical grade and used without any purification.

Cell lines. The mouse pancreatic $\beta$ cells line NIT-1 was gifted from Prof Morahan (The University of Western Australia). 


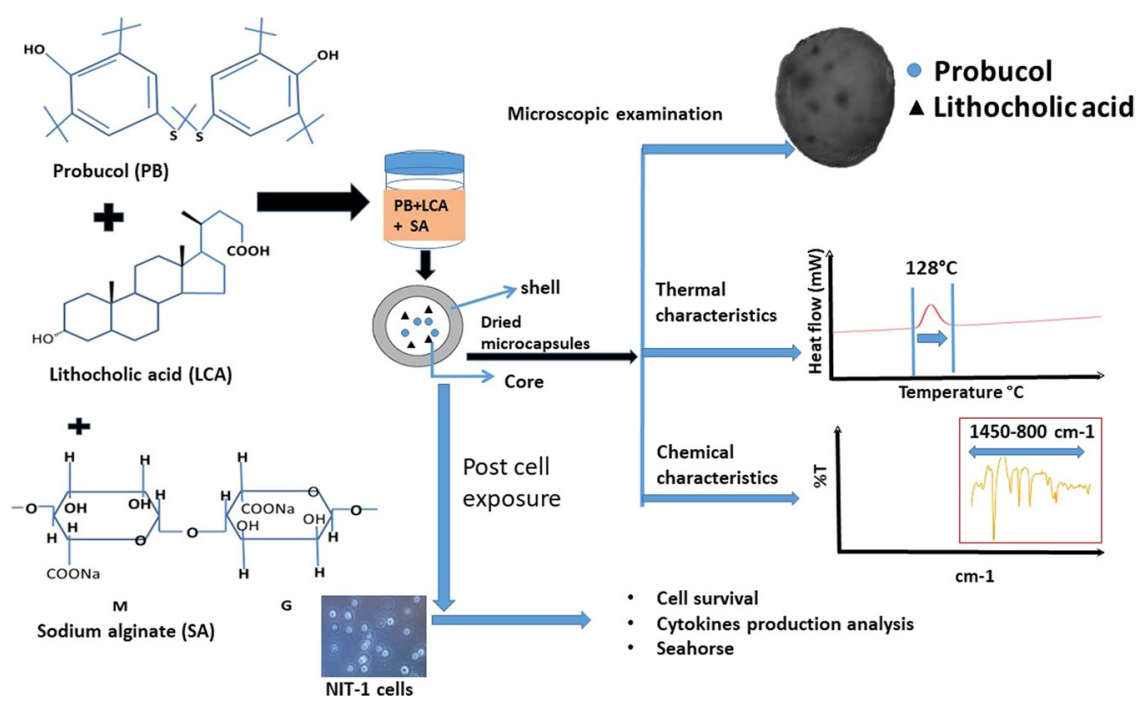

Figure 6. The summary figure of the study.

Drug preparations. Drug stock suspension of PB $(2.5 \mathrm{mg} / \mathrm{ml})$ and LCA $(1 \mathrm{mg} / \mathrm{ml})$ were made by adding powder with $5 \%$ ultra-water-soluble gel. The $2 \% \mathrm{w} / \mathrm{v} \mathrm{CaCl}_{2}$ standard solution was prepared by mixing $\mathrm{CaCl}_{2}$ with deionized water. All chemical and solvents were thoroughly mixed for 10 hours at room temperature, kept in the fridge and processed within 48 hours of preparation.

Microcapsules production. PB-SA and PB-LCA-SA loaded low viscosity SA microcapsule were prepared by using ionic gelation vibrational jet flow technique (BUCHI Labortechnik, Switzerland), in a constant ratio of 1:30 and 1:3:30 respectively. This proportion was based upon the previous publications ${ }^{9,10}$. The parameters were fixed in a frequency range of $1500-2000 \mathrm{~Hz}$ with a constant air pressure of 350 mbar with a flow rate of $5 \mathrm{ml} /$ minute. All formulations and microcapsules (PB-SA and PB-LCA-SA) were made, and three independent lots of microcapsules were produced $(\mathrm{n}=3)$.

Characterization of loaded microcapsules. Morphology, size analysis and chemical characterization of microcapsules. All microcapsules (both formulations) were prepared and analysed within 48 hours. The morphological analysis and size of microcapsules were undertaken by using OM, SEM and EDS. The microcapsules' diameters were calculated with the help of the software ToupTek which is provided within the instrument.

Briefly, 20 freshly prepared microcapsules were dried and randomly taken to access the morphological characteristics and microcapsules' diameter using an optical microscope (Nikon SM2800, Japan mounted with Toup-view Photonics, Co., Ltd Hangzhou, China).

SEM (Neon 40EsB FIB-SEM; Zeiss, Oberkochen, Germany) was used to measure the surface morphology of microcapsules. Multiple pictures from different angles and multiple scales were performed to capture the details of the surface topography. To interpret atoms distribution present in microcapsules, was obtained by using EDS (INCA X-Act; Oxford Instruments, UK). Before analysis, dried microcapsules were mounted on a glass stub and coated under vacuum ${ }^{10}$.

Determination of rheological parameters. Rheological parameters including viscosity, shear stress and torque of both formulations were done for freshly made mixtures (prior to gelation), using $2 \mathrm{ml}$ aliquots $(\mathrm{n}=3)$ at room temperature (Visco-88 viscometer, Malvern Instruments, Malvern, UK).

Thermal analysis. Thermal analysis was undertaken by DSC (DSC 8000, PerkinElmer Inc., Waltham, MA, USA). Five mg of $\mathrm{PB}$ powder or its microcapsules were loaded into a sealed pan and heated at $30{ }^{\circ} \mathrm{C}$ per minute at a flow rate of $20 \mathrm{ml} / \mathrm{minutes}$ under nitrogen in the 30- 250 range. For reference (control), an empty aluminium pan was utilised.

Chemical stability studies. FTIR was deployed to determine the chemistry profiles of each formulation and the prepared microcapsules. The spectra of drug, their physical mixtures and formulated microcapsules were measured by FTIR spectrometer-TWO (PerkinElmer Inc., Waltham, MA, USA) in transmission in the frequency range $450-4000 \mathrm{~cm}^{-1}$.

Microcapsule disintegration, drug release and dissolution measurements. Two and a half grams of microcapsules were weighed and suspended in $100 \mathrm{ml}$ of simulated intestinal fluids at four different $\mathrm{pH}$ values of $1.5,3,6$ and 7.8 at $37^{\circ} \mathrm{C}$. The sink condition was maintained throughout the experiment, and the dissolution medium was stirred at $200 \mathrm{rpm}$ for $6 \mathrm{hrs}^{10}$. PB concentrations were measured with a UV spectrophotometer (Schimadzu UV-Vis spectrophotometer 1240, Japan) at $242 \mathrm{~nm}$ using our published methods ${ }^{10}$. To exclude any 
interferences and ensure only $\mathrm{PB}$ was being measured at this particular wavelength, microcapsules without drug (SA microcapsules) were also analysed in all four $\mathrm{pH}$ values. The study was carried out in triplicate $(\mathrm{n}=3)$.

Pancreatic NIT-1 $\beta$ cells biological examination. The cells were stored in liquid nitrogen and were cultured on T-75 $\mathrm{cm}^{2}$ flasks (Thermo Fisher Scientific, Australia) with Dulbecco's Eagle Medium (DMEM) (Sigma-Aldrich, USA) supplemented with $10 \%$ foetal bovine serum (Thermo Fisher Scientific, Australia), 5.5 mmol glucose (Sigma-Aldrich, USA) and $1 \%$ penicillin streptomycin (Gibco, Life Technologies, USA ${ }^{58}$.

The MTT assays (3- (4, 5 - dimethylthiazol-2-yl)-2, 5- diphenyltetrazolium bromide) was used to determine cellular viability of NIT-1 cells after exposure with microcapsules at two glucose concentration $(5.5 \mathrm{mmol}$ and $25 \mathrm{mmol}$ ) over 48 hours. The MTT stock solution $(5 \mathrm{mg} / \mathrm{ml}$ ) (Sigma Chemical CO, USA) was prepared using phosphate buffer at pH 7.4 (Thermo Fisher Scientific, Australia). A well-established method was used to determine the cellular viability of microcapsules treated NIT- 1 cells ${ }^{8,58,59}$. Briefly, after 48 hours of incubation, microcapsules were removed from the 96 wells plates (Thermo Fisher Scientific, Australia) that have been placed in $200 \mu \mathrm{l}$ of media (pH 7.4) and $20 \mu \mathrm{l}$ of MTT from the prepared stock solution were added into each 96-wells plates. After 4 hours, MTT conversion to formazan was stopped by adding DMSO (Sigma Chemical CO, USA). The MTT assay was performed by using microplate spectrophotometer system (PerkinElmer Multimode Plate Readers, USA) at $550 \mathrm{~nm}$.

The evaluation of mitochondrial activities of microcapsules treated NIT-1 cells were done in real-time using an in-house developed method with Seahorse Flux Analyser XF 96 (Seahorse Bioscience, USA) ${ }^{8}$.

The level of cytokines (pro-inflammatory and anti-inflammatory) production were measured to test the effect of PB loaded microcapsules on treated NIT-1 cells. NIT-1 cells were cultured in DMEM medium with microcapsules at glucose concentration of $5.5 \mathrm{mmol}$ and $25 \mathrm{mmol}$ for 48 hours and microcapsules were removed and aliquots of the media were tested for IL-10, IL-1 beta, and IFN- gamma via cytokine bead array flow cytometric analysis (BD Bioscience cytometric Bead Array Mouse, USA $)^{8,10}$ using cell analyzer BD FACSCanto II (BD Bioscience, USA). Data analysis was carried out using the computer software FlowJO (FlowJo, Ashland, Oregon).

Statistical analysis. Graph Pad Prism version X8.2 (Graphpad, Inc., USA) was used to create graph and results are presented as mean \pm SD. Statistical measurements were carried out using parametric/non-parametric analysis or using a one way ANOVA and a Tuckey post-hoc, as appropriate set the level of significance.

Received: 2 September 2019; Accepted: 1 April 2020;

Published online: 07 May 2020

\section{References}

1. Lipinski, C. A., Lombardo, F., Dominy, B. W. \& Feeney, P. J. Experimental and computational approaches to estimate solubility and permeability in drug discovery and development settings1. Advanced drug delivery reviews 46, 3-26 (2001).

2. Pouton, C. W. Lipid formulations for oral administration of drugs: non-emulsifying, self-emulsifying and 'self-microemulsifying'drug delivery systems. European Journal of Pharmaceutical Sciences 11, S93-S98 (2000).

3. Humberstone, A. J. \& Charman, W. N. Lipid-based vehicles for the oral delivery of poorly water soluble drugs. Advanced drug delivery reviews 25, 103-128 (1997).

4. Singh, M., Hemant, K., Ram, M. \& Shivakumar, H. Microencapsulation: A promising technique for controlled drug delivery. Research in pharmaceutical sciences 5, 65 (2010).

5. Gupta, S., Kesarla, R. \& Omri, A. Formulation strategies to improve the bioavailability of poorly absorbed drugs with special emphasis on self-emulsifying systems. ISRN pharmaceutics 2013 (2013).

6. Negrulj, R., Mooranian, A. \& Al-Salami, H. Potentials and limitations of bile acids in type 2 diabetes mellitus: applications of microencapsulation as a novel oral delivery system. Journal of Endocrinology and Diabetes Mellitus 1, 49-59 (2013).

7. Mooranian, A., Negrulj, R. \& Al-Salami, H. The incorporation of water-soluble gel matrix into bile acid-based microcapsules for the delivery of viable beta-cells of the pancreas, in diabetes treatment: biocompatibility and functionality studies. Drug Deliv Transl Res 6, 17-23, https://doi.org/10.1007/s13346-015-0268-5 (2016).

8. Mooranian, A. et al. Advanced bile acid-based multi-compartmental microencapsulated pancreatic $\beta$-cells integrating a polyelectrolyte-bile acid formulation, for diabetes treatment. Artificial cells, nanomedicine, and biotechnology 44, 588-595 (2016).

9. Mathavan, S., Chen-Tan, N., Arfuso, F. \& Al-Salami, H. A comprehensive study of novel microcapsules incorporating gliclazide and a permeation enhancing bile acid: hypoglycemic effect in an animal model of Type-1 diabetes. Drug delivery 23, 2869-2880 (2016).

10. Mooranian, A. et al. An advanced microencapsulated system: a platform for optimized oral delivery of antidiabetic drug-bile acid formulations. Pharmaceutical development and technology 20, 702-709 (2015).

11. Mathavan, S., Chen-Tan, N., Arfuso, F. \& Al-Salami, H. The role of the bile acid chenodeoxycholic acid in the targeted oral delivery of the anti-diabetic drug gliclazide, and its applications in type 1 diabetes. ArtifCells Nanomed Biotechnol 44, 1508-1519, https://doi. org/10.3109/21691401.2015.1058807 (2016).

12. van der Walle, C. F., Sharma, G. \& Ravi Kumar, M. Current approaches to stabilising and analysing proteins during microencapsulation in PLGA. Expert opinion on drug delivery 6, 177-186 (2009).

13. Mooranian, A. et al. Stability and biological testing of taurine-conjugated bile acid antioxidant microcapsules for diabetes treatment. Ther Deliv 10, 99-106, https://doi.org/10.4155/tde-2018-0034 (2019).

14. Mooranian, A. et al. Pharmacological effects of nanoencapsulation of human-based dosing of probucol on ratio of secondary to primary bile acids in gut, during induction and progression of type 1 diabetes. ArtifCells Nanomed Biotechnol 46, S748-s754, https:// doi.org/10.1080/21691401.2018.1511572 (2018).

15. Mooranian, A. et al. Alginate-combined cholic acid increased insulin secretion of microencapsulated mouse cloned pancreatic beta cells. Ther Deliv 8, 833-842, https://doi.org/10.4155/tde-2017-0042 (2017).

16. Organization, W. H. International statistical classification of diseases and related health problems. Vol. 1 (World Health Organization, 2004).

17. Ogurtsova, K. et al. IDF Diabetes Atlas: Global estimates for the prevalence of diabetes for 2015 and 2040. Diabetes research and clinical practice 128, 40-50 (2017)

18. Wild, S., Roglic, G., Green, A., Sicree, R. \& King, H. Global prevalence of diabetes: estimates for the year 2000 and projections for 2030. Diabetes care 27, 1047-1053 (2004). 
19. Chen, L., Magliano, D. J. \& Zimmet, P. Z. The worldwide epidemiology of type 2 diabetes mellitus—present and future perspectives. Nature Reviews Endocrinology 8, 228 (2012).

20. Franco, O. H., Steyerberg, E. W., Hu, F. B., Mackenbach, J. \& Nusselder, W. Associations of diabetes mellitus with total life expectancy and life expectancy with and without cardiovascular disease. Archives of internal medicine 167, 1145-1151 (2007).

21. Garces, F. et al. High plasma phospholipase A2 activity, inflammation markers, and LDL alterations in obesity with or without type 2 diabetes. Obesity (Silver Spring) 18, 2023-2029, https://doi.org/10.1038/oby.2010.9 (2010).

22. Icks, A. et al. Drug costs in prediabetes and undetected diabetes compared with diagnosed diabetes and normal glucose tolerance: results from the population-based KORA Survey in Germany. Diabetes Care 36, e53-54, https://doi.org/10.2337/dc12-0997 (2013).

23. Assmann, G., Schulte, H., von Eckardstein, A. \& Huang, Y. High-density lipoprotein cholesterol as a predictor of coronary heart disease risk. The PROCAM experience and pathophysiological implications for reverse cholesterol transport. Atherosclerosis 124, S11-S20 (1996).

24. Cai, D. (Oxford University Press, 2012).

25. Karpe, F., Dickmann, J. R. \& Frayn, K. N. Fatty acids, obesity, and insulin resistance: time for a reevaluation. Diabetes 60, 2441-2449 (2011).

26. Yamashita, S. \& Matsuzawa, Y. Where are we with probucol: a new life for an old drug? Atherosclerosis 207, 16-23 (2009).

27. Heel, R., Brogden, R., Speight, T. \& Avery, G. Probucol: A review of its pharmacological properties and therapeutic use in patients with hypercholesterolaemia. Drugs 15, 409-428 (1978).

28. Whelehan, M. \& Marison, I. W. Microencapsulation using vibrating technology. J Microencapsul 28, 669-688, https://doi.org/10.31 09/02652048.2011.586068 (2011)

29. Lalic-Popovic, M. et al. Deoxycholic Acid as a Modifier of the Permeation of Gliclazide through the Blood Brain Barrier of a Rat. J Diabetes Res 2013, 598603, https://doi.org/10.1155/2013/598603 (2013)

30. Mooranian, A. et al. The biological effects of the hypolipidaemic drug probucol microcapsules fed daily for 4 weeks, to an insulinresistant mouse model: potential hypoglycaemic and anti-inflammatory effects. Drug delivery and translational research 8, 543-551 (2018).

31. Mooranian, A. et al. Bile acid bio-nanoencapsulation improved drug targeted-delivery and pharmacological effects via cellular flux: 6-months diabetes preclinical study. Scientific Reports 10, 106, https://doi.org/10.1038/s41598-019-53999-1 (2020).

32. Mooranian, A. et al. Novel nano-encapsulation of probucol in microgels: scanning electron micrograph characterizations, buoyancy profiling, and antioxidant assay analyses. Artif Cells Nanomed Biotechnol 46, S741-s747, https://doi.org/10.1080/21691401.2018.15 11571 (2018).

33. Mooranian, A. et al. Eudragit((R))-based microcapsules of probucol with a gut-bacterial processed secondary bile acid. Ther Deliv 9, 811-821, https://doi.org/10.4155/tde-2018-0036 (2018).

34. Mooranian, A. et al. Probucol release from novel multicompartmental microcapsules for the oral targeted delivery in type 2 diabetes. Aaps Pharmscitech 16, 45-52, https://doi.org/10.1208/s12249-014-0205-9 (2015).

35. Zhao, M.-X., Cai, Z.-C., Zhu, B.-J. \& Zhang, Z.-Q. The apoptosis effect on liver cancer cells of gold nanoparticles modified with lithocholic acid. Nanoscale research letters 13, 1-8 (2018).

36. Sreekanth, V. et al. Tethering of chemotherapeutic drug/imaging agent to bile acid-phospholipid increases the efficacy and bioavailability with reduced hepatotoxicity. Bioconjugate chemistry 28, 2942-2953 (2017).

37. Patil, S. et al. Novel self-assembled lithocholic acid nanoparticles for drug delivery in cancer. RSC Advances 3, 19760-19764 (2013).

38. Ward, J. B. J. et al. Ursodeoxycholic acid and lithocholic acid exert anti-inflammatory actions in the colon. American Journal of Physiology-Gastrointestinal and Liver Physiology 312, G550-G558, https://doi.org/10.1152/ajpgi.00256.2016 (2017).

39. Mooranian, A., Negrulj, R., Arfuso, F. \& Al-Salami, H. Characterization of a novel bile acid-based delivery platform for microencapsulated pancreatic beta-cells. Artif Cells Nanomed Biotechnol 44, 194-200, https://doi.org/10.3109/21691401.2014.9344 57 (2016).

40. Davignon, J. In Principles and Treatment of Lipoprotein Disorders 429-469 (Springer, 1994).

41. Awasthi, R. \& Kulkarni, T. G. Development of novel gastroretentive floating particulate drug delivery system of gliclazide. Current drug delivery $9,437-451$ (2012).

42. Prajapati, S., Tripathi, P., Ubaidulla, U. \& Anand, V. Design and development of gliclazide mucoadhesive microcapsules: in vitro and in vivo evaluation. Aaps Pharmscitech 9, 224 (2008).

43. Pamies, R. \& Schmidt, R. R. Martínez, M. d. C. L. \& de la Torre, J. G. The influence of mono and divalent cations on dilute and nondilute aqueous solutions of sodium alginates. Carbohyd Polym 80, 248-253 (2010).

44. Legrand, J., Dumont, E., Comiti, J. \& Fayolle, F. Diffusion coefficients of ferricyanide ions in polymeric solutions-comparison of different experimental methods. Electrochimica acta 45, 1791-1803 (2000).

45. Soares, J., Santos, J., Chierice, G. \& Cavalheiro, E. Thermal behavior of alginic acid and its sodium salt. Eclética Química 29, 57-64 (2004).

46. Thybo, P., Pedersen, B. L., Hovgaard, L., Holm, R. \& Müllertz, A. Characterization and physical stability of spray dried solid dispersions of probucol and PVP-K30. Pharmaceutical development and technology 13, 375-386 (2008).

47. Kulkarni, A. R., Soppimath, K. S., Aminabhavi, T. M. \& Rudzinski, W. E. In-vitro release kinetics of cefadroxil-loaded sodium alginate interpenetrating network beads. Eur J Pharm Biopharm 51, 127-133 (2001).

48. Ajun, W., Yan, S., Li, G. \& Huili, L. Preparation of aspirin and probucol in combination loaded chitosan nanoparticles and in vitro release study. Carbohyd Polym 75, 566-574 (2009).

49. Mooranian, A. et al. An optimized probucol microencapsulated formulation integrating a secondary bile acid (deoxycholic acid) as a permeation enhancer. Drug design, development and therapy 8, 1673 (2014).

50. Mathavan, S., Mikov, M., Golocorbin-Kon, S. \& Al-Salami, H. Diabetes development increased concentrations of the conjugated bile acid, taurocholic acid in serum, while treatment with microencapsulated-taurocholic acid exerted no hypoglycaemic effects. Eur J Pharm Sci 106, 1-9, https://doi.org/10.1016/j.ejps.2017.05.041 (2017).

51. Mooranian, A., Negrulj, R., Arfuso, F. \& Al-Salami, H. The effect of a tertiary bile acid, taurocholic acid, on the morphology and physical characteristics of microencapsulated probucol: potential applications in diabetes: a characterization study. Drug delivery and translational research 5, 511-522 (2015).

52. Al-Salami, H. et al. Probiotic Pre-treatment Reduces Gliclazide Permeation (ex vivo) in Healthy Rats but Increases It in Diabetic Rats to the Level Seen in Untreated Healthy Rats. Archives of drug information 1,35-41 (2008).

53. Gorogawa, S.-i et al. Probucol preserves pancreatic $\beta$-cell function through reduction of oxidative stress in type 2 diabetes. Diabetes research and clinical practice 57, 1-10 (2002).

54. Liu, J. H., Liu, D. F., Wang, N. N., Lin, H. L. \& Mei, X. Possible role for the thioredoxin system in the protective effects of probucol in the pancreatic islets of diabetic rats. Clinical and Experimental Pharmacology and Physiology 38, 528-533 (2011).

55. Brand, M. D. \& Nicholls, D. G. Assessing mitochondrial dysfunction in cells. Biochemical Journal 435, 297-312 (2011).

56. Wikstrom, J. D. et al. A novel high-throughput assay for islet respiration reveals uncoupling of rodent and human islets. PloS one 7 , e33023 (2012).

57. Mooranian, A., Tackechi, R., Jamieson, E., Morahan, G. \& Al-Salami, H. Innovative Microcapsules for Pancreatic beta-Cells Harvested from Mature Double-Transgenic Mice: Cell Imaging, Viability, Induced Glucose-Stimulated Insulin Measurements and Proinflammatory Cytokines Analysis. Pharm Res 34, 1217-1223, https://doi.org/10.1007/s11095-017-2138-y (2017). 
58. Mooranian, A., Negrulj, R. \& Al-Salami, H. The influence of stabilized deconjugated ursodeoxycholic acid on polymer-hydrogel system of transplantable NIT-1 cells. Pharmaceutical research 33, 1182-1190 (2016).

59. Mathavan, S., Chen-Tan, N., Arfuso, F. \& Al-Salami, H. Morphological, Stability, and Hypoglycemic Effects of New Gliclazide-Bile Acid Microcapsules for Type 1 Diabetes Treatment: the Microencapsulation of Anti-diabetics Using a Microcapsule-Stabilizing Bile Acid. Aaps Pharmscitech 19, 3009-3018, https://doi.org/10.1208/s12249-018-1127-8 (2018).

\section{Acknowledgements}

The use of laboratory equipment, scientific and technical assistance of Microscopy and Microanalysis Facility at Curtin University which has been partially funded by the University, State and Commonwealth Governments. The work is partially supported by the European Union's Horizon 2020 research and innovation programme under the Marie Skłodowska-Curie grant agreement No. 872370. The authors acknowledge the project for Scientific and Technological Development of Vojvodina No. 114-451-2072-/2016-02.

\section{Author contributions}

The study was designed by Al-Salami, Mikov, Golocorbin-Kon, Mooranian and analysis and interpretation of research data was done by Wagle, Walker, Kovacevic and Gedawy. Drafting significant parts of the work or critically revising it, which contributes to the interpretation was done by all authors, significant contribution to data interpretation and presentation resulting in significant improvement of quality was done by all authors; providing data or analytical skills, which add significantly to the design, quality and readability of the work was done also by all the authors.

\section{Competing interests}

Al-Salami H. has been and is currently receiving funding from Beijing Nat-Med Biotechnology Co. Ltd.

\section{Additional information}

Correspondence and requests for materials should be addressed to H.A.-S.

Reprints and permissions information is available at www.nature.com/reprints.

Publisher's note Springer Nature remains neutral with regard to jurisdictional claims in published maps and institutional affiliations.

(c) (i) Open Access This article is licensed under a Creative Commons Attribution 4.0 International License, which permits use, sharing, adaptation, distribution and reproduction in any medium or format, as long as you give appropriate credit to the original author(s) and the source, provide a link to the Creative Commons license, and indicate if changes were made. The images or other third party material in this article are included in the article's Creative Commons license, unless indicated otherwise in a credit line to the material. If material is not included in the article's Creative Commons license and your intended use is not permitted by statutory regulation or exceeds the permitted use, you will need to obtain permission directly from the copyright holder. To view a copy of this license, visit http://creativecommons.org/licenses/by/4.0/.

(c) The Author(s) 2020 